\title{
Supplementary Information: \\ Pnicogen Bonds Pairing an Anionic Lewis Acid with Neutral and Anionic Bases
}

\section{Steve Scheiner, ${ }^{* 1}$ Rafał Wysokiński, ${ }^{2}$ Mariusz Michalczyk ${ }^{2}$ and Wiktor Zierkiewicz ${ }^{* 2}$}

${ }^{1}$ Department of Chemistry and Biochemistry, Utah State University Logan, Utah 84322-0300, United States

${ }^{2}$ Faculty of Chemistry, Wrocław University of Science and Technology, Wybrzeże

Wyspiańskiego 27, 50-370 Wrocław, Poland

Table S1. Structural parameters (distances in $\AA$, angles in degs) of $\mathrm{ZCl}_{4}{ }^{-}$monomers calculated at the MP2/aug-cc-pVDZ level of theory.

\begin{tabular}{|l|c|c|l|l|l|l|l|l|}
\hline & \multicolumn{4}{|c|}{ gas phase } & \multicolumn{5}{c|}{ aqueous } \\
\hline & $\mathrm{r}(\mathrm{Z}-\mathrm{Cl1})$ & $\mathrm{r}(\mathrm{Z}-\mathrm{Cl} 2)$ & $\mathrm{Cl1-Z-Cl1}$ & $\mathrm{Cl2-Z-C12}$ & $\mathrm{r}(\mathrm{Z}-\mathrm{Cl1})$ & $\mathrm{r}$ (Z-C12) & C11-Z-C11 & $\mathrm{C} 12-\mathrm{Z}-\mathrm{C} 12$ \\
$\mathrm{PCl}_{4}^{-}$ & 2.406 & 2.123 & 174.7 & 99.5 & $2.159,2.990$ & 2.105 & 172.7 & 99.3 \\
\hline $\mathrm{AsCl}_{4}^{-}$ & 2.474 & 2.232 & 174.6 & 98.5 & 2.485 & 2.229 & 177.7 & 98.1 \\
\hline $\mathrm{SbCl}_{4}^{-}$ & 2.600 & 2.416 & 179.8 & 97.2 & 2.616 & 2.424 & 175.2 & 95.6 \\
\hline
\end{tabular}

Table S2. AIM descriptors of the of $\mathrm{ZCl}_{4}^{-}(\mathrm{Z}=\mathrm{P}, \mathrm{As}, \mathrm{Sb})$ monomers. Bond critical point (BCP) properties: electron density $\rho$, Laplacian of electron density $\nabla^{2} \rho$ and total electron energy $\mathrm{H}$, were obtained at the MP2/ aug-cc-pVDZ level. Data in atomic units.

\begin{tabular}{|l|l|l|l|l|l|l|l|}
\hline & \multicolumn{3}{|c|}{ gas phase } & \multicolumn{2}{l|}{ aqueous solution } \\
\hline & interaction & $\rho$ & $\nabla^{2} \rho$ & $\mathrm{H}$ & $\rho$ & $\nabla^{2} \rho$ & $\mathrm{H}$ \\
\hline $\mathrm{PCl}_{4}^{-}$ & $\mathrm{P}-\mathrm{Cl} 1$ & 0.111 & -0.108 & -0.078 & 0.114 & -0.107 & -0.084 \\
\cline { 2 - 8 } & $\mathrm{P}-\mathrm{Cl} 2$ & 0.068 & +0.034 & -0.023 & 0.105 & -0.088 & 0.068 \\
\hline $\mathrm{AsCl}_{4}^{-}$ & $\mathrm{As}-\mathrm{Cl} 1$ & 0.096 & +0.057 & -0.045 & 0.097 & +0.056 & -0.046 \\
\cline { 2 - 8 } & $\mathrm{As}-\mathrm{Cl} 2$ & 0.062 & +0.072 & -0.016 & 0.060 & +0.072 & -0.015 \\
\hline $\mathrm{SbCl}_{4}^{-}$ & $\mathrm{Sb}-\mathrm{Cl} 1$ & 0.074 & +0.133 & -0.022 & 0.073 & +0.132 & -0.021 \\
\cline { 2 - 8 } & $\mathrm{Sb}-\mathrm{Cl} 2$ & 0.054 & +0.094 & -0.011 & 0.052 & +0.091 & -0.011 \\
\hline
\end{tabular}


Table S3. Deformation energies $\left(\mathrm{E}_{\mathrm{def}}, \mathrm{kcal} / \mathrm{mol}\right)$ of complexes with $\mathrm{ZCl}_{4}{ }^{-}$calculated at the MP2/aug-cc-pVDZ level.

\begin{tabular}{|l|c|c|c|c|c|c|c|c|c|c|}
\hline & $\mathrm{ZCl}_{4}^{-}$ & pyr & total & $\mathrm{ZCl}_{4}^{-}$ & $\mathrm{NCH}$ & total & $\mathrm{ZCl}_{4}^{-}$ & $\mathrm{CN}^{-}$ & total \\
\hline & \multicolumn{3}{|c|}{ pyr } & \multicolumn{3}{c|}{$\mathrm{NCH}$} & \multicolumn{3}{c|}{$\mathrm{CN}^{-}$} \\
\hline $\mathrm{PCl}_{4}^{-}$ & 39.07 & 1.47 & 40.54 & 32.69 & 0.18 & 32.87 & 45.61 & 0.19 & 45.80 \\
\hline $\mathrm{AsCl}_{4}^{-}$ & 31.89 & 1.45 & 33.34 & 25.49 & 0.13 & 25.62 & 39.49 & 0.20 & 39.69 \\
\hline $\mathrm{SbCl}_{4}^{-}$ & 21.72 & 1.10 & 22.82 & 15.52 & 0.09 & 15.61 & 31.91 & 0.18 & 32.09 \\
\hline
\end{tabular}

Table S4. AIM descriptors of the of complexes of bases with $\mathrm{ZCl}_{4}{ }^{-}$. Bond critical point (BCP) properties: electron density $\rho$, Laplacian of electron density $\nabla^{2} \rho$ and total electron energy $\mathrm{H}$, were obtained at the MP2/ aug-cc-pVDZ level. Data in atomic units.

\begin{tabular}{|l|l|c|c|c|c|c|c|}
\hline \multirow{2}{*}{} & \multirow{2}{*}{ interaction } & \multicolumn{3}{|c|}{ pyridine } & \multicolumn{3}{c|}{$\mathrm{NCH}$} \\
\cline { 2 - 8 } & & $\rho$ & $\nabla^{2} \rho$ & $\mathrm{H}$ & $\rho$ & $\nabla^{2} \rho$ & $\mathrm{H}$ \\
\hline $\mathrm{PCl}_{4}^{-}$ & $\mathrm{P}-\mathrm{N}$ & 0.100 & -0.028 & -0.067 & 0.087 & 0.187 & -0.045 \\
\cline { 2 - 8 } & $\mathrm{P}-\mathrm{Cl}$ & 0.071 & +0.027 & -0.025 & 0.074 & 0.002 & -0.029 \\
\cline { 2 - 8 } & $\mathrm{H}-\mathrm{Cl}$ & 0.017 & +0.061 & +0.002 & & & \\
\hline $\mathrm{AsCl}_{4}^{-}$ & $\mathrm{As}-\mathrm{N}$ & 0.091 & +0.117 & -0.037 & 0.070 & 0.195 & -0.020 \\
\cline { 2 - 8 } & $\mathrm{As}-\mathrm{Cl}$ & 0.063 & +0.067 & -0.017 & 0.067 & 0.063 & -0.020 \\
\cline { 2 - 8 } & $\mathrm{H}-\mathrm{Cl}$ & 0.016 & +0.054 & +0.002 & & & \\
\hline $\mathrm{SbCl}_{4}^{-}$ & $\mathrm{Sb}-\mathrm{N}$ & 0.070 & +0.169 & -0.016 & 0.046 & 0.170 & -0.003 \\
\cline { 2 - 8 } & $\mathrm{Sb}-\mathrm{Cl}$ & 0.054 & +0.090 & -0.012 & 0.057 & 0.100 & -0.012 \\
\cline { 2 - 8 } & $\mathrm{H}-\mathrm{Cl}$ & 0.014 & +0.047 & +0.001 & & & \\
\hline
\end{tabular}

Table S5. AIM descriptors of the of complexes of $\mathrm{CN}^{-}$anion with $\mathrm{ZCl}_{4}{ }^{-}$. Bond critical point (BCP) properties: electron density $\rho$, Laplacian of electron density $\nabla^{2} \rho$ and total electron energy $\mathrm{H}$, were obtained at the MP2/ aug-cc-pVDZ level. Data in atomic units.

\begin{tabular}{|l|l|l|l|r|}
\hline \multirow{2}{*}{} & \multirow{2}{*}{ interaction } & \multicolumn{3}{|c|}{$\mathrm{CN}^{-}$} \\
\cline { 3 - 5 } & & \multicolumn{1}{|c|}{$\rho$} & \multicolumn{1}{|c|}{$\nabla^{2} \rho$} & $\mathrm{H}$ \\
\hline $\mathrm{PCl}_{4}{ }^{-}$ & $\mathrm{P}-\mathrm{C}$ & 0.139 & 0.137 & -0.107 \\
\cline { 2 - 5 } & $\mathrm{P}-\mathrm{Cl}$ & 0.062 & 0.039 & -0.018 \\
\hline $\mathrm{AsCl}_{4}^{-}$ & $\mathrm{As}-\mathrm{C}$ & 0.128 & 0.044 & -0.082 \\
\cline { 2 - 5 } & $\mathrm{As}-\mathrm{Cl}$ & 0.056 & 0.065 & -0.013 \\
\hline $\mathrm{SbCl}_{4}^{-}$ & $\mathrm{Sb}-\mathrm{C}$ & 0.094 & 0.172 & -0.035 \\
\cline { 2 - 5 } & $\mathrm{Sb}-\mathrm{Cl}$ & 0.047 & 0.079 & -0.009 \\
\hline
\end{tabular}




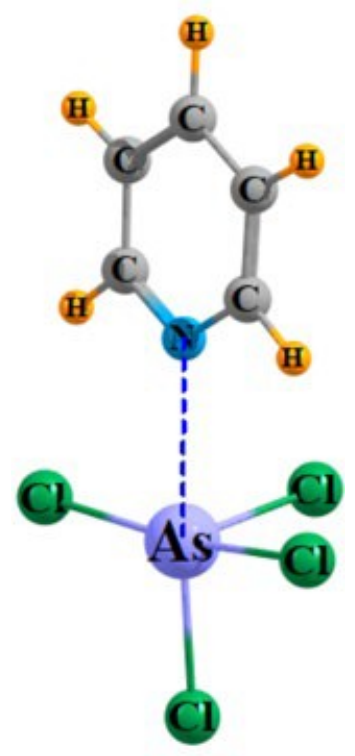

Fig. S1. Secondary minimum on the potential energy surface of $\mathrm{pyr}^{\cdots}{ }^{\cdots} \mathrm{ZCl}_{4}{ }^{-}$. 

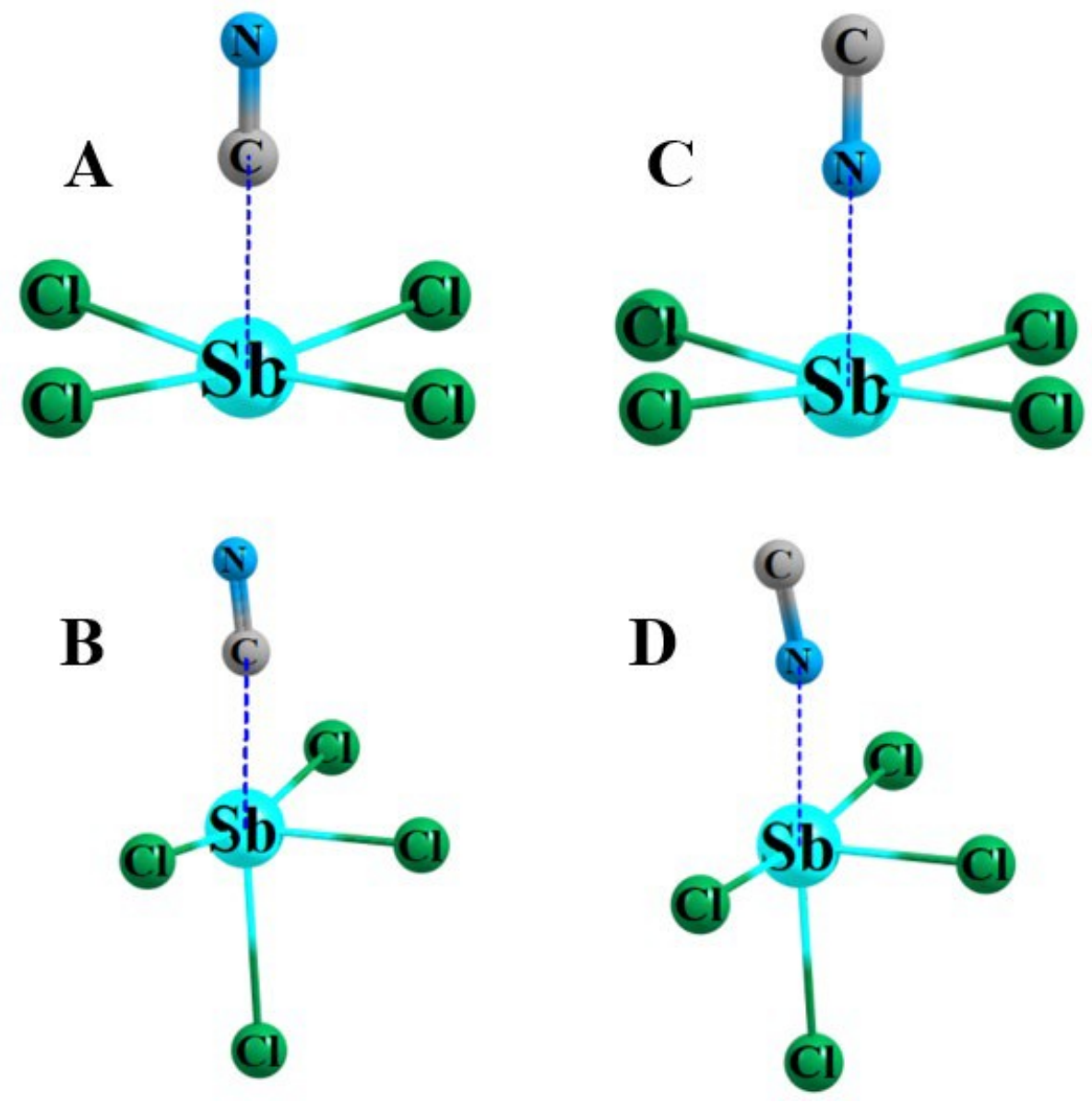

Fig. S2. Minima on the potential energy surface of $\mathrm{CN}^{-\cdots} \mathrm{ZCl}_{4}{ }^{-}$.

Table S6. Electronic and Gibbs free energies $\mathrm{E}_{\mathrm{el}}$ and $\mathrm{G}(298 \mathrm{~K})(\mathrm{kcal} / \mathrm{mol})$ of $\mathrm{NC}^{-} \cdots \mathrm{ZCl}_{4}{ }^{-}$ conformers relative to global minimum A, calculated at the MP2/aug-cc-pVDZ level of theory.

\begin{tabular}{|l|c|c|c|c|c|c|}
\hline & \multicolumn{3}{|c|}{$\mathrm{E}_{\text {el }}$} & \multicolumn{3}{c|}{$\Delta \mathrm{G}$} \\
\hline & $\mathrm{B}$ & $\mathrm{C}$ & $\mathrm{D}$ & $\mathrm{B}$ & $\mathrm{C}$ & $\mathrm{D}$ \\
\hline $\mathrm{NC}^{-\cdots} \mathrm{PCl}_{4}^{-}$ & - & 18.51 & 21.94 & - & 18.22 & 19.64 \\
\hline $\mathrm{NC}^{-\cdots} \mathrm{AsCl}_{4}^{-}$ & 7.96 & 16.12 & 18.87 & 6.60 & 15.74 & 17.57 \\
\hline $\mathrm{NC}^{-\cdots} \mathrm{SbCl}_{4}^{-}$ & 7.55 & 10.95 & 12.80 & 6.63 & 10.58 & 11.66 \\
\hline
\end{tabular}


Table S7. Structural parameters (distances in $\AA$, angles in degs) for the complexes studied in aqueous solution calculated at the MP2/aug-cc-pVDZ level.

\begin{tabular}{|c|c|c|c|c|}
\hline & $R(Z \cdots N)$ & $\mathrm{r}(\mathrm{Z}-\mathrm{Cl})$ & $\theta(\mathrm{N} \cdots \mathrm{Z}-\mathrm{Cl})$ & $\theta(\mathrm{Cl}-\mathrm{Z}-\mathrm{Cl})$ \\
\hline & \multicolumn{4}{|c|}{ pyridine } \\
\hline $\mathrm{pyr}^{\prime} \cdots \mathrm{PCl}_{4}^{-}$ & 1.943 & 2.402 & 87.4 & 89.9 \\
\hline $\mathrm{pyr}^{\prime} \cdot \mathrm{AsCl}_{4}^{-}$ & 2.051 & 2.474 & 86.3 & 89.7 \\
\hline \multirow[t]{2}{*}{ pyr $^{\cdots} \mathrm{SbCl}_{4}^{-}$} & 2.243 & 2.617 & 82.4 & 89.0 \\
\hline & \multicolumn{4}{|c|}{$\mathrm{NCH}$} \\
\hline $\mathrm{HCN} \cdots \mathrm{PCl}_{4}^{-}$ & 1.883 & 2.379 & 83.9 & 167.8 \\
\hline $\mathrm{HCN} \cdots \mathrm{AsCl}_{4}$ & 2.036 & 2.452 & 82.7 & 165.4 \\
\hline \multirow[t]{3}{*}{$\mathrm{HCN}^{\cdots} \mathrm{SbCl}_{4}$} & 2.281 & 2.592 & 78.8 & 157.6 \\
\hline & \multicolumn{4}{|c|}{$\mathrm{CN}^{-}$} \\
\hline & $R(Z \cdots C)$ & $\mathrm{r}(\mathrm{Z}-\mathrm{Cl})$ & 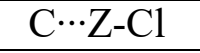 & $\mathrm{Cl}-\mathrm{Z}-\mathrm{Cl}$ \\
\hline${ }^{-} \mathrm{NC}^{\prime} \cdots \mathrm{PCl}_{4}^{-}$ & 1.828 & $2.450^{\mathrm{a}}$ & $86.4^{\mathrm{a}}$ & $89.8^{\mathrm{a}}$ \\
\hline${ }^{-} \mathrm{NC} \cdots \mathrm{AsCl}_{4}{ }^{-}$ & 1.946 & 2.507 & 85.7 & 89.7 \\
\hline${ }^{-} \mathrm{NC}^{\prime \cdots \mathrm{SbCl}_{4}}{ }^{-}$ & 2.163 & 2.649 & 83.3 & 89.2 \\
\hline
\end{tabular}

${ }^{a}$ average value

Table S8. Structural parameters (distances in $\AA$, angles in degs) for the most stable cis configurations (Fig 3) of $\mathrm{HCN}^{\cdots} \mathrm{ZCl}_{4}{ }^{-}$in aqueous solution calculated at the MP2/aug-cc-pVDZ level.

\begin{tabular}{|c|c|c|c|c|c|}
\hline & $\mathrm{R}(\mathrm{Z} \cdots \mathrm{N})$ & $\mathrm{r}\left(\mathrm{Z}-\mathrm{Cl}_{\mathrm{a}}\right)$ & $\mathrm{r}\left(\mathrm{Z}-\mathrm{Cl} \mathrm{b}_{\mathrm{b}}\right)$ & $\mathrm{r}\left(\mathrm{Z}-\mathrm{Cl}_{\mathrm{c}}\right)$ & $\theta(\mathrm{Z} \cdots \mathrm{N}-\mathrm{C})$ \\
\hline $\mathrm{HCN} \cdots \mathrm{PCl}_{4}{ }^{-}$ & 3.132 & $\begin{array}{l}2.977 \\
2.166\end{array}$ & 2.111 & 2.105 & 108.8 \\
\hline $\mathrm{HCN} \cdots \mathrm{AsCl}_{4}^{-}$ & 2.883 & 2.495 & 2.253 & 2.232 & 172.2 \\
\hline $\mathrm{HCN} \cdots \mathrm{SbCl}_{4}^{-}$ & 2.843 & 2.631 & 2.461 & 2.427 & 175.9 \\
\hline
\end{tabular}


Table S9. AIM descriptors of the of complexes of trans and cis complexes of $\mathrm{HCN}$ with $\mathrm{ZCl}_{4}{ }^{-}$in water. Bond critical point (BCP) properties: electron density $\rho$, Laplacian of electron density $\nabla^{2} \rho$ and total electron energy H, were obtained at the MP2/ aug-cc-pVDZ level. Data in atomic units.

\begin{tabular}{|l|l|c|c|c|c|c|c|}
\hline \multirow{2}{*}{} & \multirow{2}{*}{ interaction } & \multicolumn{3}{|c|}{ trans } & \multicolumn{3}{|c|}{ cis } \\
\cline { 2 - 8 } & & $\rho$ & $\nabla^{2} \rho$ & $\mathrm{H}$ & $\rho$ & $\nabla^{2} \rho$ & $\mathrm{H}$ \\
\hline $\mathrm{PCl}_{4}{ }^{-}$ & $\mathrm{P}-\mathrm{N}$ & 0.099 & 0.157 & -0.059 & 0.010 & 0.030 & 0.001 \\
\cline { 2 - 8 } & ${\mathrm{P}-\mathrm{Cl}^{\mathrm{a}}}^{2}$ & 0.073 & 0.020 & -0.026 & 0.090 & -0.052 & -0.062 \\
\hline $\mathrm{AsCl}_{4}{ }^{-}$ & $\mathrm{As}-\mathrm{N}$ & 0.086 & 0.193 & -0.032 & 0.017 & 0.051 & 0.001 \\
\cline { 2 - 8 } & $\mathrm{As}-\mathrm{Cl}^{\mathrm{a}}$ & 0.065 & 0.065 & -0.019 & 0.078 & 0.061 & -0.030 \\
\hline $\mathrm{SbCl}_{4}{ }^{-}$ & $\mathrm{Sb}-\mathrm{N}$ & 0.059 & 0.194 & -0.009 & 0.022 & 0.057 & -0.000 \\
\cline { 2 - 8 } & $\mathrm{Sb}-\mathrm{Cl}^{\mathrm{a}}$ & 0.055 & 0.090 & -0.012 & 0.061 & 0.113 & -0.014 \\
\hline
\end{tabular}

${ }^{a}$ average of all four $\mathrm{Z}-\mathrm{Cl}$ quantities 